\title{
UCRL-ID-128656
}

\section{Modeling Electron Heat Transport During Magnetic Field Buildup in SSPX}

D. D. Hua, E. B. Hooper, T. K. Fowler

October, 1997

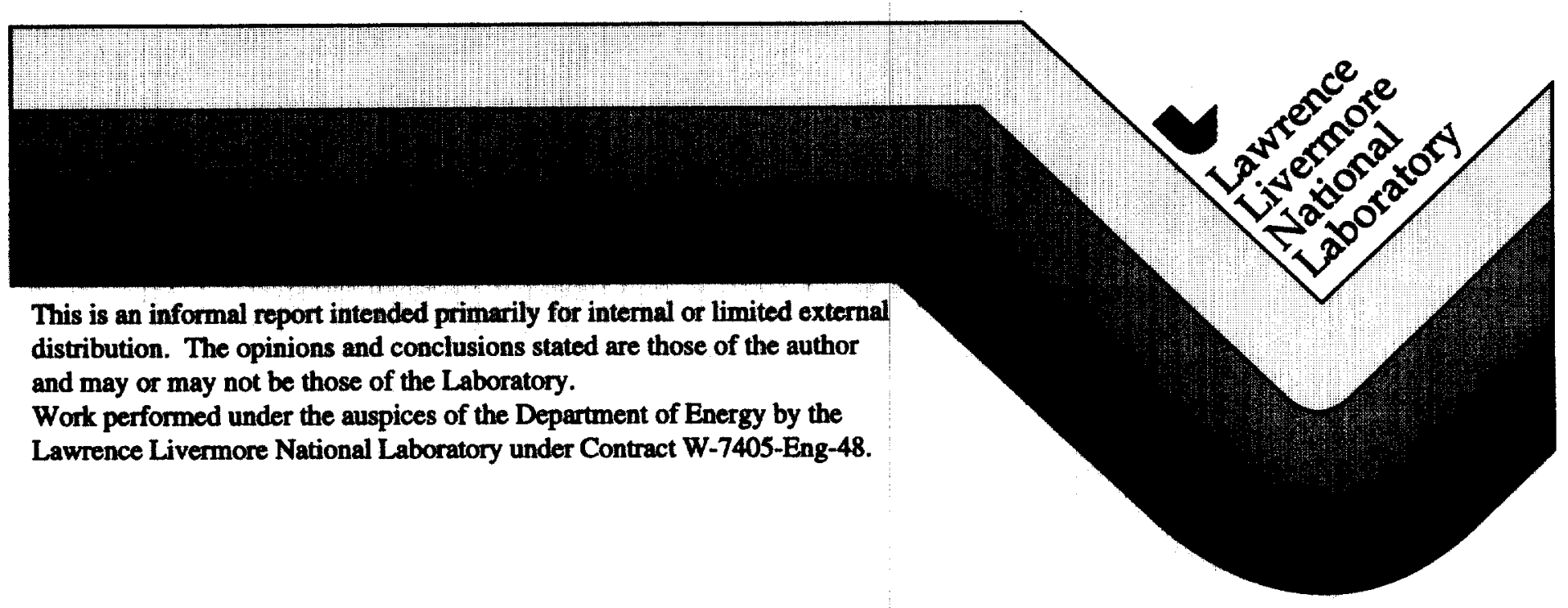




\section{DISCLAIMER}

This document was prepared as an account of work sponsored by an agency of the United States Government. Neither the United States Government nor the Univernity of Califomin nor any of their employees, makes any warranty, express or implied, or assumes any legal lisbility or responsibility for the accuracy, completeness, or usefulness of any information, apparatus, product, or process disclosed, or represents that its use would not infringe privately owned rights. Reference herein to any specific commercial product, process, or service by trade name, trademark, manufacturer, or otherwise, does not necessarily constitute or imply its endorsement, recommendation, or favoring by the United States Government or the University of California. The views and opinions of authors expressed herein do not necessarily state or reflect those of the United States Government or the University of California, and shall not be used for advertising or product endorsement purposes.

This report has been reproduced directly from the best available copy.

Available to DOE and DOE contractors from the Office of Scientific and Technical Information P.O. Box 62, Oak Ridge, TN 37831

Prices available from (615) 576-8401, FTS 626-8401

Availeble to the public from the

National Technical Information Service

L. L. Rua, U.S. Department of Commerce

October, 1997

This is an informal report intended primarily for internal or limited external distribution. The opinions and conclusions stated are those of the author and may or may not be those of the Laboratory.

Work performed under the auspices of the Department of Energy by the Lawrence Livermore National Laboratory under Contract W-7405-Eng-48. 


\title{
Modeling Electron Heat Transport During Magnetic Field Buildup in SSPX
}

\author{
D. D. Hua, ${ }^{a}$ E. B. Hooper ${ }^{b}$ and T. K. Fowler ${ }^{a}$ \\ ${ }^{a}$ Department of Nuclear Engineering \\ University of California \\ Berkeley, CA 94720 \\ ${ }^{b}$ Lawrence Livermore National Laboratory \\ University of California \\ Livermore, CA 94551
}

\begin{abstract}
A model for spheromak magnetic field buildup and electron thermal transport, including a thermal diffusivity associated with magnetic turbulence during helicity injection [1], is applied to a SSPX equilibrium, with a maximum final magnetic field of 1.3 T. Magnetic field-buildup times of $1.0 \times 10^{-3}, 5.0 \times 10^{-4}$ and $1.0 \times 10^{-4} \mathrm{~s}$ were used in the model to examine their effects on electron thermal transport. It is found that at transport run time of $4 \times 10^{-3} \mathrm{~s}$, the fastest buildup-time results in the highest final temperature profile, with a core temperature of $0.93 \mathrm{keV}$ while requiring the lowest input energy at $140 \mathrm{KJ}$. The results show that within the model the most rapid buildup rate generates the highest electron temperature at the fastest rate and at the lowest consumption of energy. However, the peak power requirements are large ( $>600 \mathrm{MW}$ for the fastest buildup case examined), suggesting that effects surh as imnurity oonoration. whirh lis outside the modal mav lasd to a rato whirh
\end{abstract}




\section{Introduction}

The following model, in cylindrical coordinates and calibrated to CTX [1], is applied to the SSPX,

$$
\begin{aligned}
\frac{\partial}{\partial t}\left[\frac{3}{2} n T\right]+\frac{1}{r} \frac{\partial}{\partial r}\left[r n \chi\left(\frac{\partial T}{\partial r}\right)\right] & =\eta j^{2} \\
\frac{\partial}{\partial t}\left[\frac{B^{2}}{2 \mu}\right]+\frac{1}{r} \frac{\partial}{\partial r}[r P(r)] & =-\eta j^{2},
\end{aligned}
$$

where Eq. (1) is the temperature diffusion equation and Eq. (2) the magnetic energy diffusion equation. Here all quantities are expressed in MKS units except for $T$, which is in $k \mathrm{eV}$. In the equations, $n(r)$ is the electron density (in units of $\left.10^{20} \mathrm{~m}^{-3}\right), T(r, t)$ the electron temperature, $B(r, t)$ the magnetic field, and $P(r)= \pm\left(v_{A} \tilde{B}^{2} / \mu\right)$ the Poynting vector (representing helicity injection that drives the turbulent magnetic field $\tilde{B}$ ) where $v_{A}=1.63 \times 10^{6}(B / \sqrt{n})[\mathrm{m} / \mathrm{s}]$ is the Alfvén speed. In addition, $\eta j^{2}$ is the Ohmic heating where $\eta=\left(4 \times 10^{-8}\right) T^{-\frac{3}{2}}\left[W /\left(m^{3} A^{2}\right)\right]$ is the Spitzer resistivity and $j=(\lambda B / \mu)$ is the current density, obtained from Maxwell's equations and the Taylor state [2], assumed in this model. The diffusion coefficient in Eq. (1) is

$$
\chi=\chi_{G B}+\chi_{m a g}
$$

where

$$
\begin{aligned}
\chi_{G B} & =\left[\frac{0.6 T^{\frac{3}{2}}}{B^{2} a}\right], \\
\chi_{m a g} & =v_{e} L_{c}\left[\frac{\tilde{B}}{B}\right]^{2} .
\end{aligned}
$$

$\chi_{G B}$ is an approximate Gyrobohm diffusion coefficient arising from electrostatic turbulence, and $\chi_{m a g}$ is the Rechester-Rosenbluth coefficient arising from magnetic turbulence [3]. In Eqs. (3) and (4) $a$ is the minor radius, $v_{e}=1.88 \times 10^{7} \sqrt{T}[\mathrm{~m} / \mathrm{s}]$ the electron thermal speed, and $L_{c}=3.2 a$ from fitting previous results to CTX data [1,4]. Manipulating Eq. (2) to get $\tilde{B}$ in terms of other quantities $[1],{ }^{1}$ we arrive at

\footnotetext{
${ }^{1}$ we have modeled the spheromak as a cylinder with length $(2 \pi a)$ and radius $a$.
} 


$$
\chi_{m a g}=\frac{v_{e} L_{c} \mu}{v_{A} B^{2}}\left[\frac{P_{\Omega}(r)}{(2 \pi)^{2} a r}\right] g_{p}(r)
$$

where

$$
\begin{aligned}
& P_{\Omega}(r)=\int d V \eta j^{2} \\
& g_{p}(r)=\left|\left[\frac{\int^{r} d V B^{2}}{\int^{a} d V B^{2}}\right] \frac{\int^{a} d V \frac{\partial}{\partial t}\left[\frac{B^{2}}{2 \mu}\right]}{P_{\Omega}(r)}+1\right|=\left|\left\lceil\frac{\int^{r} d V B^{2}}{\int^{a} d V B^{2}}\right] \frac{P_{m}}{P_{\Omega}(r)}+1\right| .
\end{aligned}
$$

In Eq. (6), $P_{\Omega}(r)$ is the partial Ohmic power $\left(P_{\Omega}(a)\right.$ is the total Ohmic power. For the rest of this paper, $P_{\Omega}$ means the total Ohmic power) and $P_{m} \equiv \int^{a} d V \frac{\partial}{\partial t}\left[\frac{B^{2}}{2 \mu}\right]$ is the magnetic power, defined as that part of the input power spent in building up the magnetic field. Hence $\left[g_{p}(a)-1\right]$ is the ratio of magnetic power to Ohmic power. As explained in Ref. [1], Eqs. (5) and (6) are derived by integrating Eq. (2) in $r$ assuming $B(r)$ is of constant shape representative of the Taylor state and using this to derive $\tilde{B}^{2}$ appearing in $\chi_{\text {mag. }}$. It is this procedure that gives rise to the form factor appearing in Eq. (6).

\section{Corsica Results for SSPX}

The diffusion coefficients defined in Eqs. (3) and (5) are substituted into the transport module of corsica [5], a general plasma equilibrium and transport code written in flux variables with effective minor radius $\bar{\rho}$ given by

$$
\bar{\rho}=\frac{\psi}{\psi_{\max }}
$$

with $\psi$ being the toroidal flux coordinate. In addition, the magnetic field $B(\bar{\rho}, t) \equiv \sqrt{\left\langle B^{2}\right\rangle}$ where $\langle\cdot\rangle$ represents the flux surface average. To utilize our model described in Section 1 in corsica, the integration limits in Eq. (6) become $\bar{\rho}$ and in the denominator of Eq. (5) we set $r=a \bar{\rho}^{\frac{1}{2}}$, representative of the behavior of $\psi$ near the origin.

The prescribed magnetic field and its time-derivative are

$$
B(\bar{\rho}, t)=B_{i}(\bar{\rho})+\left[B_{f}(\bar{\rho})-B_{i}(\bar{\rho})\right]\left(1-e^{-\frac{t}{\tau}}\right)
$$




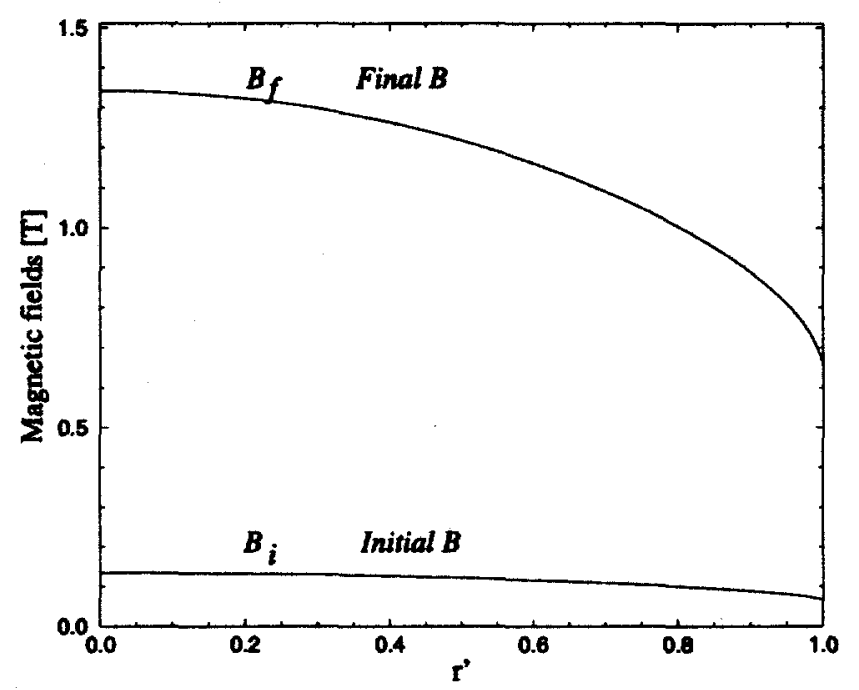

Figure 1: Initial and final magnetic field profiles.

$$
\dot{B}(\bar{\rho}, t)=\frac{1}{\tau}\left[B_{f}(\bar{\rho})-B_{i}(\bar{\rho})\right] e^{-\frac{\varepsilon}{\tau}} .
$$

where $B_{i}(\bar{\rho})$ is the initial field and has an arithmetic mean of $0.1 T$ and $B_{f}(\bar{\rho})$ is the final field and has a mean of $1.0 T . B_{i}(\bar{\rho})$ and $B_{f}(\bar{\rho})$, by construction, have the same spatial profile. Fig. 1 plots $B_{i}$ and $B_{f}$ versus $r^{\prime}$, defined as

$$
r^{\prime}=\bar{\rho}^{\frac{1}{2}}
$$

In all subsequent figures, spatial profiles are plotted in the $r^{\prime}$ variable. In Eqs. (7) and (8), $\tau$ is the characteristic field buildup time. The following buildup times, $\tau_{1}=1.0 \times 10^{-3}$, $\tau_{2}=5.0 \times 10^{-4}$ and $\tau_{3}=1.0 \times 10^{-4} s$ are used in the corsica runs. The transport run time is set to $4.0 \times 10^{-3} s$, approximately the proposed SSPX magnetic gun time.

The initial temperature profile is $T_{i}(\bar{\rho})=0.02 \mathrm{keV}$, a flat profile, and the boundary condition is $T(\bar{\rho}=1)=0.02 \mathrm{keV} .{ }^{2}$ Also, the density and $q$ profiles, plotted in Fig. 2, are kept constant during the transport run.

The time-evolution of the thermal diffusivities, temperature, energy and other quantities

${ }^{2}$ The other boundary condition, $\left.\frac{d T}{d r}\right|_{r=0}=0$ is automatically satisfied if $\left.\frac{d T}{d \bar{\phi}}\right|_{\bar{\rho}=0}$ is finite. 


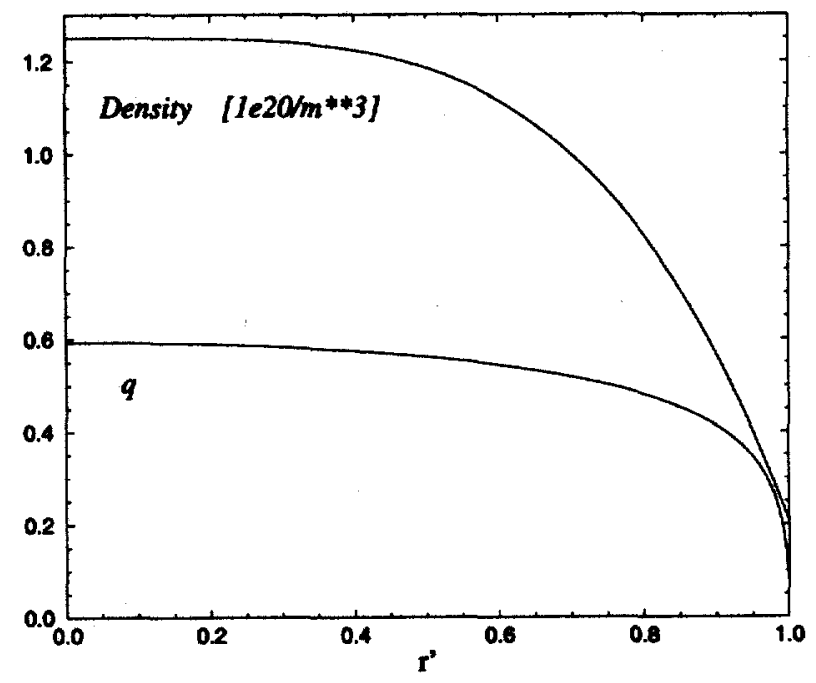

Figure 2: $q$ and density profile of a SSPX equilibrium.

of interest are presented in the following sections. For convenience, quantities associated with $\tau_{1}, \tau_{2}$ and $\tau_{3}$ will be subscripted 1,2 and 3 respectively.

\subsection{Thermal Diffusivity}

Fig. 3 plots the initial and final $\chi$ profiles for $\tau_{1}, \tau_{2}$ and $\tau_{3}$. The initial temperature, at $0.02 \mathrm{keV}$, is low, thus $\chi_{G B}$ is much smaller than $\chi_{m a g}$ and could be ignored, except at $r^{\prime}=0$ where $\chi_{m a g}=0$. Initially, $\chi_{m a g}$ is quite high due to a big $\dot{B}$ in Eq. (8) which leads to a big $g_{\mathrm{p}}$ in Eq. (6) which then leads to a big $\chi_{m a g}$ in Eq. (5). The initial $\dot{B}$ 's also cause $\chi_{3}$ to be an order of magnitude bigger than $\chi_{1}$ : since $\tau_{3} \ll \tau_{1}$, then $\dot{B}_{3} \gg \dot{B}_{1}$ from Eq. (8), which leads to $P_{m 3} \gg P_{m 1}$, and since $P_{\Omega 3}=P_{\Omega 1}$, then $g_{p 3} \gg g_{p 1}$ from Eq. (6) ( $\gg$ here means approximately an order of magnitude bigger).

The situation reverses at $t=4.0 \times 10^{-3} s$ where $\dot{B}$ becomes small as prescribed by Eq. (8) which lowers $g_{p}$ and thus $\chi_{\text {mag }}$. In fact, $\dot{B}_{3} \approx 0$ leads to $g_{p 3} \approx 1$. In contrast, $\dot{B}_{1} \neq 0$ so that $P_{m 1} \approx P_{\Omega 1}$ (see Fig. 4) which leads to $g_{p 1}>1$. Therefore $g_{p 3}<g_{p 1}$ and is the main reason that $\chi_{\text {mag } 3}<\chi_{\text {mag } 1}$.

Continuing with Fig. 3, near $r^{\prime}=0$ where $\chi_{m a g} \rightarrow 0$ and temperature is highest, $\chi_{G B} \geq \chi_{m a g}$ and becomes significant. Since the temperature profile for $\tau_{3}$ is higher than 


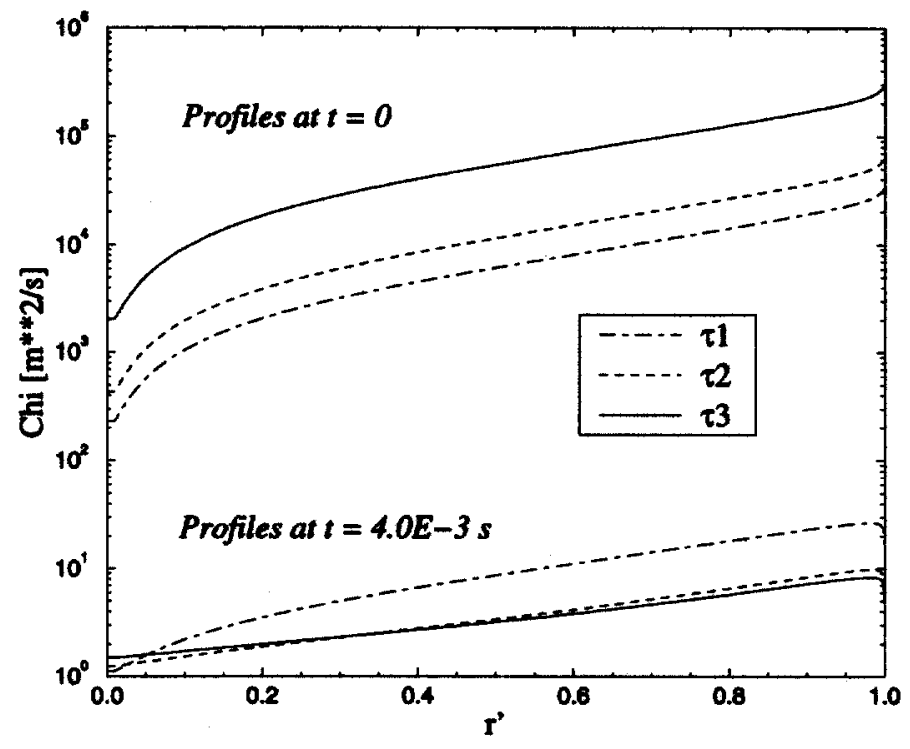

Figure 3: Initial and final $\chi$ profiles for field buildup times $\tau_{1}, \tau_{2}$ and $\tau_{3}$.

the profile for $\tau_{1}$ (see Fig. 6), then $\chi_{G B 3}>\chi_{G B 1}$. Therefore, for $0<r^{\prime}<0.05, \chi_{3}>\chi_{1}$ due to $\chi_{G B 3}>\chi_{G B 1}$ and $\chi_{m a g}$ being small, and for $0.05<r^{\prime}<1, \chi_{3}<\chi_{1}$ due to $\chi_{m a g 3}<\chi_{m a g 1}$ and $\chi_{G B}$ being small.

Fig. 4 plots the time-evolution of magnetic powers $P_{m}$ and Ohmic powers $P_{\Omega}$. From Fig. $4, P_{m 3}$ and $P_{\Omega 3}$ peak higher and earlier than $P_{m 2}$ and $P_{\Omega 2}$ respectively; which peak higher and earlier than $P_{m 1}$ and $P_{\Omega 1}$ respectively. However, $P_{m 3}$ and $P_{\Omega 3}$ also decrease faster than $P_{m 2}$ and $P_{\Omega 2}$ respectively which also in turn decrease faster than $P_{m 1}$ and $P_{\Omega 1}$.

The relative behavior of $P_{m 3}$ and $P_{m 2}$ is due to a more rapid build up and maintenance of the magnetic field for buildup time $\tau_{3}$ than $\tau_{2}$, where $B_{3}>B_{2}$ and $\dot{B}_{3}>\dot{B}_{2}$ in the beginning, but $B_{3}>B_{2}$ and $\dot{B}_{3} \ll \dot{B}_{2}$ after some elapsed time. Similarly, $B_{2}, B_{1}, \dot{B}_{2}$ and $\dot{B}_{1}$ follow the same trend to affect the relative behavior of $P_{m 2}$ and $P_{m 1}$. The relative behavior of $P_{\Omega}$ 's is due to $B_{3}>B_{2}>B_{1}$ combined with the opposite effect of $T_{3}>T_{2}>T_{1}$ (see Section 2.2).

Also from Fig. 4, $P_{m 3}<P_{\Omega 3}$ for $t>6 \times 10^{-4} s$ and rapidly approaches 0 . Similarly, $P_{m 2}<P_{\Omega 2}$ for $t>2.4 \times 10^{-3} s$ and approaches 0 . In contrast, $P_{m 1} \approx P_{\Omega 1}$ at the final time $t=4.0 \times 10^{-3} \mathrm{~s}$. The evolution of the ratio of $P_{m}$ to $P_{\Omega}$ plays a significant role in determining the effectiveness of input energy in heating the plasma (see Sect. 2.3). 


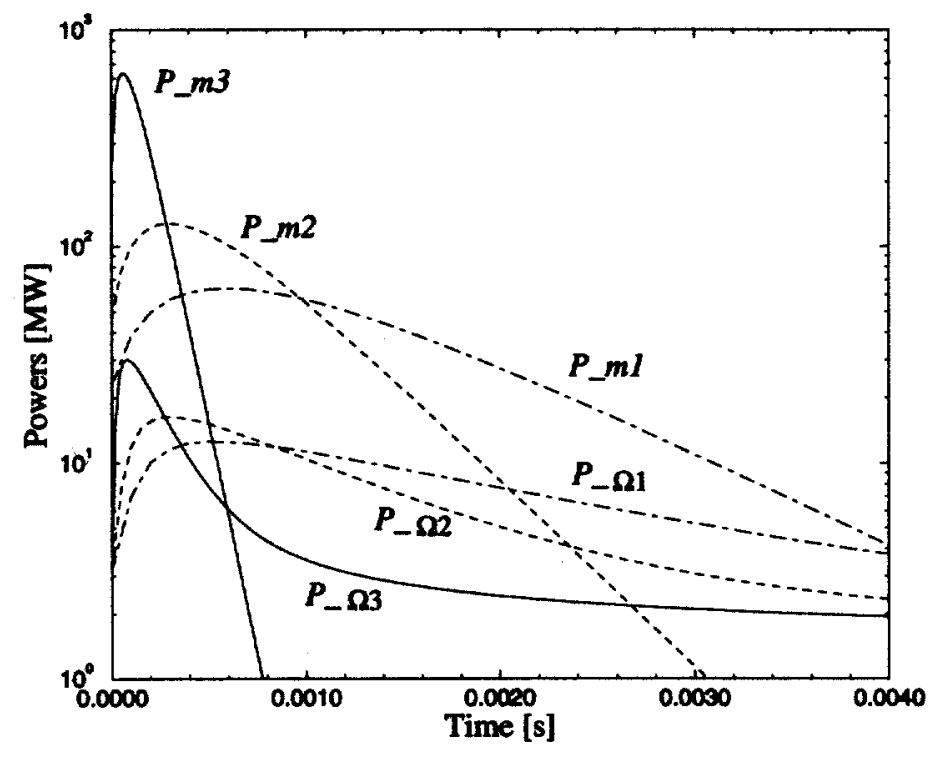

Figure 4: Time evolution of magnetic power $P_{m}$ and Ohmic power $P_{\Omega}$ for buildup times $\tau_{1}, \tau_{2}$ and $\tau_{3}$.

Defining the average $\chi$ as $\bar{\chi}=\left(\int d V \chi\right) / V$, Fig. 5 plots the time-evolution of $\bar{\chi}$ for $\tau_{1}$, $\tau_{2}$ and $\tau_{3}$. With trends similar to those in Fig. 3, $\bar{\chi}_{3}>\bar{\chi}_{2}>\bar{\chi}_{1}$ initially. But $\bar{\chi}_{3}<\bar{\chi}_{1}$ for $t>5 \times 10^{-5} s$ and $\bar{\chi}_{3}<\bar{\chi}_{2}$ for $t>3.4 \times 10^{-5} s$, while $\bar{\chi}_{2}<\bar{\chi}_{1}$ for $t>9 \times 10^{-5} \mathrm{~s}$.

\subsection{Temperature}

Fig. 6 plots the final temperature profiles for buildup times $\tau_{1}, \tau_{2}$ and $\tau_{3}$ (initial profiles are the same, all at a uniform $0.02 \mathrm{keV}$ ). The respective core temperatures are $T_{\mathrm{cl}}=0.62 \mathrm{keV}$, $T_{c 2}=0.81 \mathrm{keV}$ and $T_{c 3}=0.93 \mathrm{keV}$. Fig. 7 plots the respective normalized temperature profiles. While the profiles for $\tau_{2}$ and $\tau_{3}$ are similar, they are both flatter than that for $\tau_{1}$. The similarity of the profile for the two faster buildup rates indicates that it has reached a final shape; profile evolution is continuing for the slowest rate.

Fig. 8 plots the time evolution of the respective core temperatures, $T\left(r^{\prime}=0\right)$. As expected from Figs. 4 and 5, $T_{c 3}$ rises faster than $T_{c 2}$ which in turn rises faster than $T_{c 1}$ in the beginning due to $P_{\Omega 3}>P_{\Omega 2}>P_{\Omega 1}$ and $\bar{\chi}_{3}<\bar{\chi}_{2}<\bar{\chi}_{1}$ (for $t>5 \times 10^{-5} s$ ). However near $t=4.0 \times 10^{-3} s, T_{c 3}$ rises slower than $T_{c 2}$ which rises slower than $T_{c 1}$, because of 


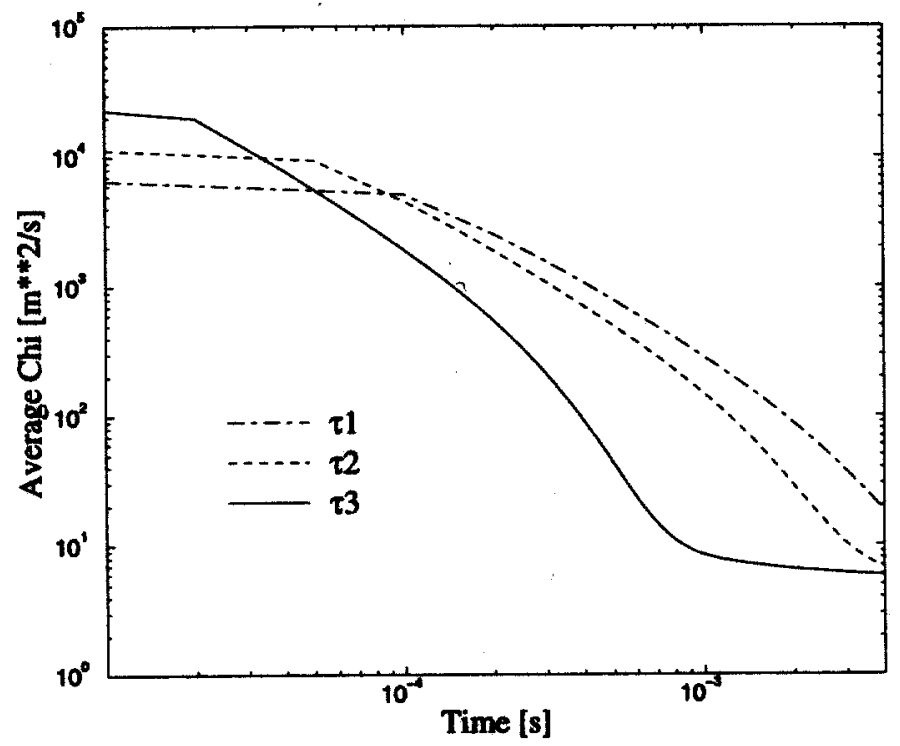

Figure 5: Time evolution of the $\bar{\chi}$ for buildup times $\tau_{1}, \tau_{2}$ and $\tau_{3}$.

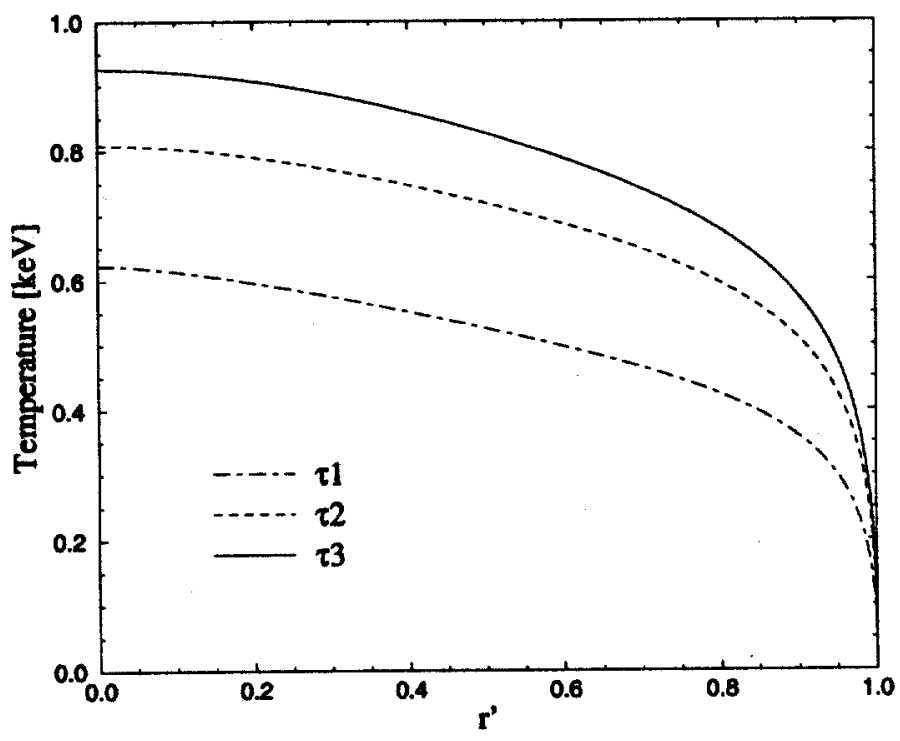

Figure 6: Final temperature profiles for buildup times $\tau_{1}, \tau_{2}$ and $\tau_{3}$. 


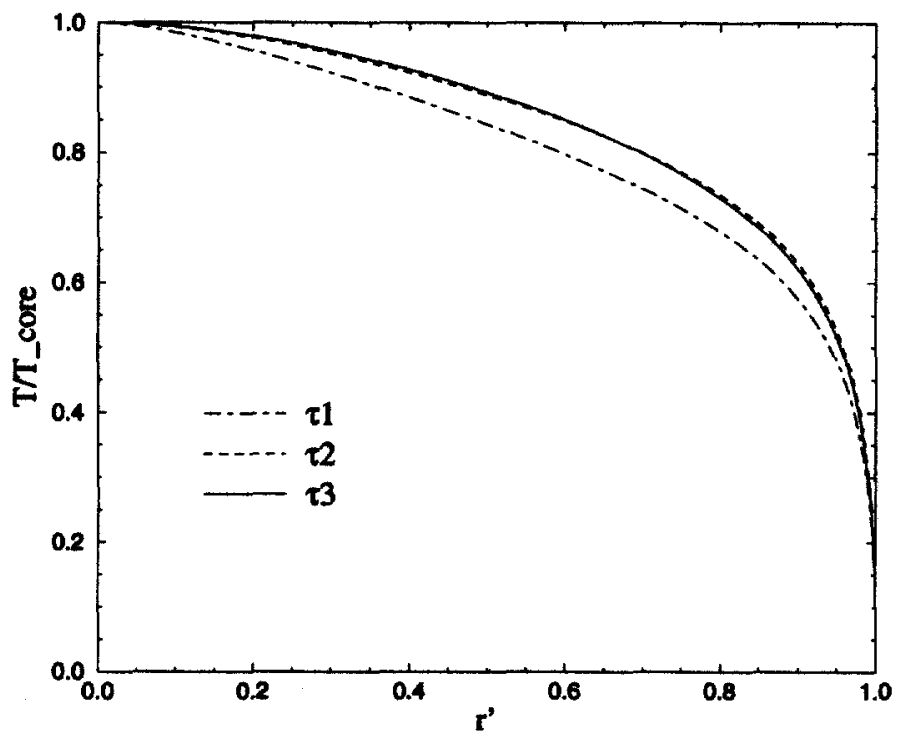

Figure 7: Normalized final temperature profiles for buildup times $\tau_{1}, \tau_{2}$ and $\tau_{3}$.

$P_{\Omega 3}<P_{\Omega 2}<P_{\Omega 1}$ despite $\bar{\chi}_{3}<\bar{\chi}_{2}<\bar{\chi}_{1}$. Within this model, we have not reached an equilibrium, steady state temperature, even for the fastest buildup rate examined.

\subsection{Energy}

Fig. 9 plots the time evolution of the input energy, defined as

$$
E_{\text {in }}=\int d t\left[P_{m}+P_{\Omega}\right]
$$

for buildup times $\tau_{1}, \tau_{2}$ and $\tau_{3}$. As expect from Fig. $4, E_{i n 3}$ rises faster and becomes nearly flat sooner than $E_{i n 2}$, which retains the same trends with respect to $E_{i n 1}$. It is interesting to note that $E_{i n 3}<E_{i n 2} \approx E_{\text {in1 }}$ at $t=4 \times 10^{-3} \mathrm{~s}$. This is due to $\left(P_{m 3}, P_{\Omega 3}\right)<\left(P_{m 2}, P_{\Omega 2}\right)$ and $\left(P_{m 1}, P_{n 1}\right)$ respectively for $t>6 \times 10^{-4} s$ from Fig. 4 , as explained in Section 2.1.

It is also of interest to determine the effectiveness of the input energy in heating the plasma. Fig. 10 plots $T_{c}$ versus $E_{\text {in }}$. It takes $150 \mathrm{KJ}$ to get a core temperature of $0.62 \mathrm{keV}$ for buildup time $\tau_{1}$, and $150 \mathrm{KJ}$ to get a core temperature of $0.81 \mathrm{keV}$ for buildup time $\tau_{2}$, and only $140 \mathrm{KJ}$ to get a core temperature $0.93 \mathrm{keV}$ for buildup time $\tau_{3}$. This is due to the effects of $B$ and $\dot{B}$ on $P_{m}, P_{\Omega}$ and $\chi$, which were explained in Section 2.1. 


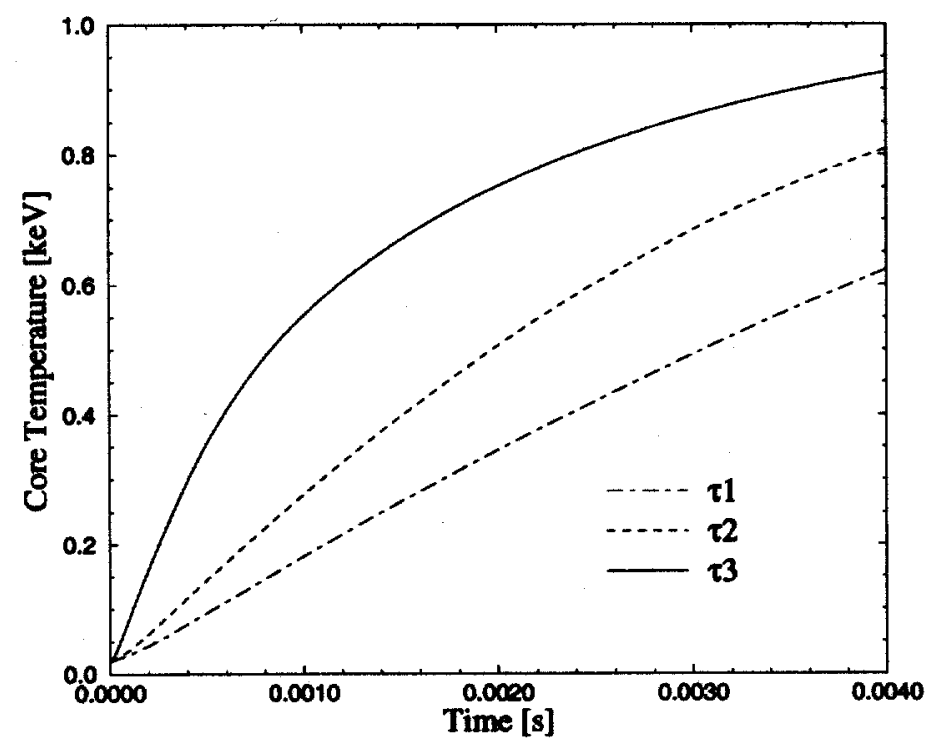

Figure 8: Time evolution of core temperature for $\tau_{1}, \tau_{2}$ and $\tau_{3}$.

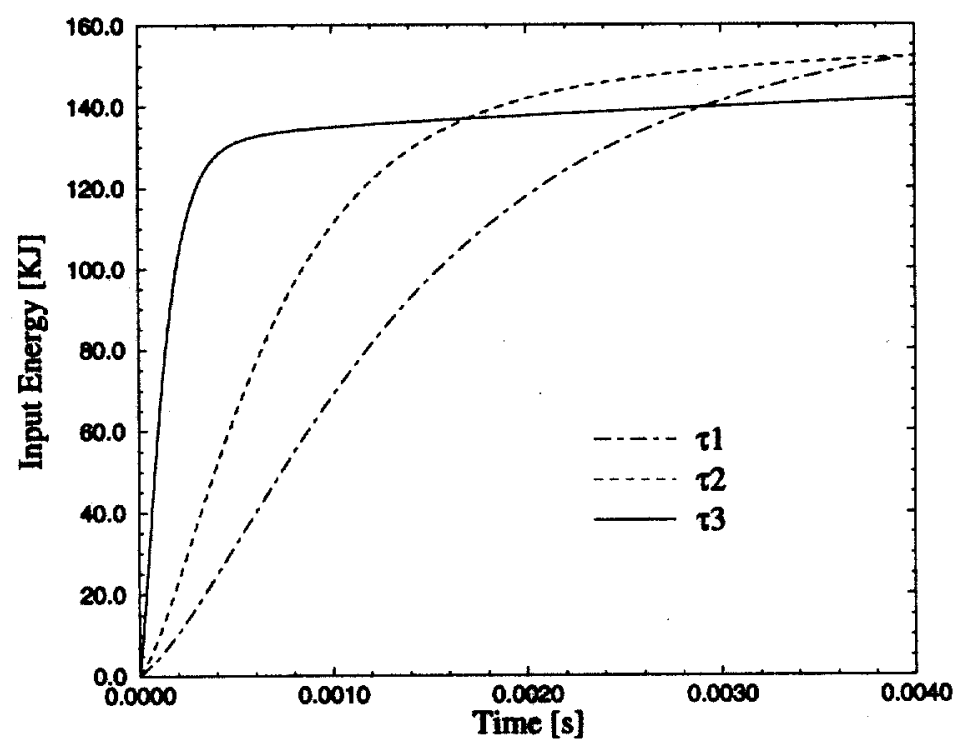

Figure 9: Time evolution of input energy for buildup times $\tau_{1}, \tau_{2}$ and $\tau_{3}$. 


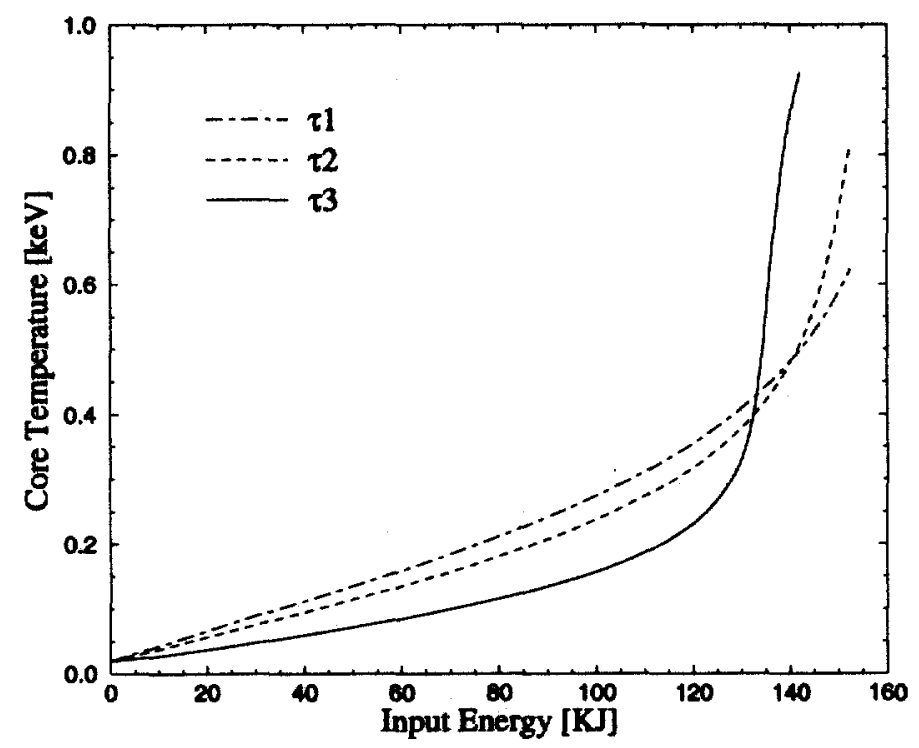

Figure 10: Core temperature versus input energy for buildup times $\tau_{1}, \tau_{2}$ and $\tau_{3}$.

For example, $T_{c 3}<T_{c 1}$ from 0 to $133 K J$ of total input energy, which corresponds to time intervals $\left[0,6.4 \times 10^{-4} s\right]$ for buildup time $\tau_{3}$ and $\left[0,2.6 \times 10^{-3} s\right]$ for buildup time $\tau_{1}$ from Fig. 9. During those times, (1) the time-averaged $\bar{\chi}_{3}=8500 \mathrm{~m}^{2} / \mathrm{s}$ whereas the time-averaged $\bar{\chi}_{1}=2700 \mathrm{~m}^{2} / \mathrm{s}$ from Fig. 5 , and (2) The Ohmic energy input is $\sim 10 \mathrm{KJ}$ for $\tau_{3}$ whereas the Ohmic energy $\sim 25 K J$ for $\tau_{1}$ from Fig. 4. Afterwards, $T_{c 3}>T_{c 1}$ from $133 \mathrm{KJ}$ onward, which corresponds to time interval $\left[6.4 \times 10^{-4} s, 4 \times 10^{-3} s\right]$ for $\tau_{3}$, and $\left[2.6 \times 10^{-3} s, 4.0 \times 10^{-3} s\right]$ for $\tau_{1}$. During those time intervals, (1) the time-averaged $\bar{\chi}_{3}=750 \mathrm{~m}^{2} / \mathrm{s}$ compared to the time-averaged $\bar{\chi}_{1}=960 \mathrm{~m}^{2} / \mathrm{s}$ and (2) the Ohmic energy input is $9 K J$ for $\tau_{3}$ compared to $6 K J$ for $\tau_{1}$. Hence, below $E_{\text {in }}=133 K J, \bar{\chi}_{3}>\bar{\chi}_{1}$ and $E_{\Omega 3}<E_{\Omega 1}$ which causes $T_{c 3}<T_{c 1}$. The situation reverses for $E_{i n}>133 K J$, which causes $T_{c 3}>T_{c 1}$.

\subsection{Other Quantities of Interests}

Using Eq. 4, Fig. 11 plots the initial and final ratio of the turbulent magnetic field to the background magnetic field, $\tilde{B} / B=\sqrt{\chi /\left(v_{e} L_{c}\right)}$. Due to the the decrease in $\chi$ (see Fig. 3) and increase in $T$ (hence an increase in $v_{e}$ ), the final ratios are about two orders of magnitude lower than the corresponding initial ratios. Moreover, these ratios have the 


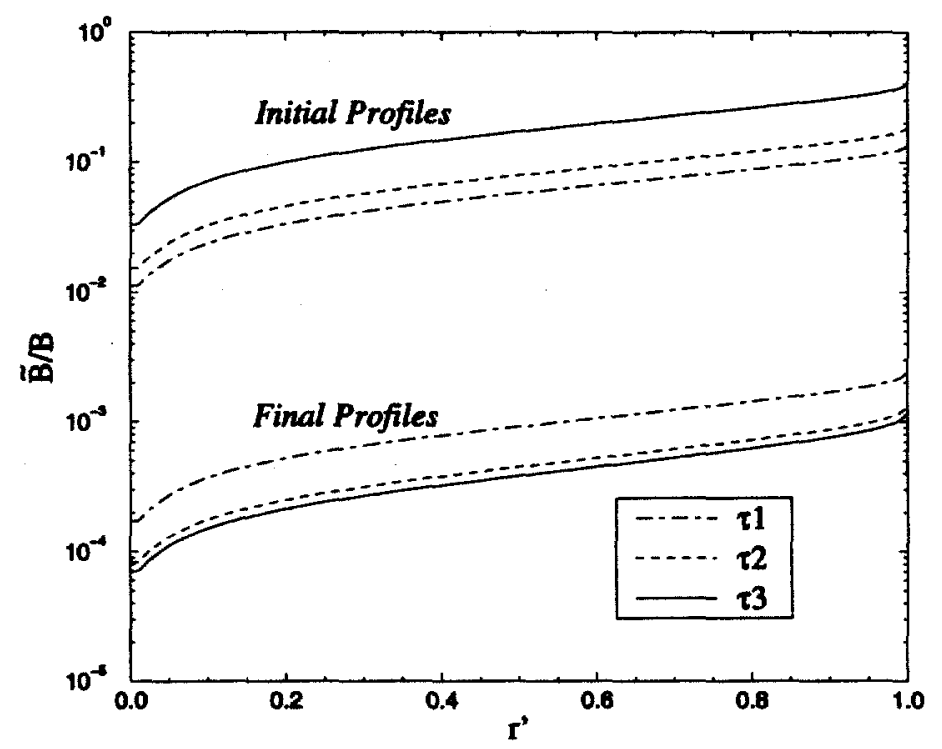

Figure 11: Initial and final $\tilde{B} / B$ for buildup times $\tau_{1}, \tau_{2}$ and $\tau_{3}$.

same characteristics as the corresponding diffusion coefficients, shown in Fig. 3: that is, the ratio with buildup time $\tau_{3}$ is the largest initially and the smallest at the end.

\section{Summary}

Using corsica's transport module with the thermal diffusivity defined in Eqs. (3) and (4), it takes $140 \mathrm{KJ}$ to heat the electron core temperature from $0.02 \mathrm{keV}$ to $0.93 \mathrm{keV}$ with a magnetic buildup time of $\tau_{3}=1.0 \times 10^{-4} s$ used in Eqs. (7) and (8). The diffusion coefficient for $\tau_{3}$, the fastest buildup time (among $\tau_{1}=1.0 \times 10^{-3} s, \tau_{2}=5 \times 10^{-4} s$ and $\tau_{3}=1.0 \times 10^{-4} \mathrm{~s}$ ), although initially the largest, rapidly becomes the smallest (on average) due mainly to the rapidly decreasing $\dot{B}_{3}$, which decreases the magnetic power $P_{m}$ and thus decreases the factor $g_{p}$ in $\chi_{m a g}$, which in the regimes of SSPX is the dominant diffusion coefficient.

Of particular interest to the experiment is the issue of optimizing the buildup rate to maximize utilization of the available energy in the electrical systems. Within the present model, if the criterion is reaching high $T_{c}$ as rapidly as possible, the rapid buildup rate is clearly the most effective. Although the thermal conductivity is very high during the initial 
buildup, the temperature is low and the thermal energy losses not so fast as to constrain the magnetic field. Then, once the field is developed and its rate of increase reduced, the radial thermal conductivity drops and the temperature increases rapidly. After $4 \mathrm{~ms}$, higher temperatures are reached at lower energy inputs than for the slower buildup rates. Note from Fig. 5 that to the extent that the goal of the experiment is to study low thermal conductivity plasmas, the fastest buildup rate exists in that state much longer than the other two rates modeled.

However, there are effects which lie outside the model and which may require optimization at a slower buildup rate. First, we note from Fig. 11 that at the fastest buildup rate, the initial magnetic field fluctuations in the model exceeds $10 \%$. At this level, the assumption that the configuration is basically one of closed magnetic surfaces with the fluctuations allowing transport without destroying the configuration may not be a good one. Certainly, the possibility that the field is so chaotic that surfaces are not a valid, zeroth-order description must be considered. If so, the entire assumptions of the model need to be re-examined. Furthermore, ion heating by the reconnection process, which has been ignored in the present calculation, is likely to be very strong leading to an energy loss channel which can be significant in an experiment.

A second concern is that wall effects, including impurity generation, may be considerably more serious for the fast buildup; these might become so bad that they prevent the achievement of a high-temperature plasma. Note from Fig. 4 that the magnetic power for the fastest buildup examined is an order-of-magnitude larger than for the slowest. In fact, for the fastest buildup the peak power exceeds $600 \mathrm{MW}$ at its peak, and inefficiencies are not included. This is probably high enough to damage the helicity injector and generate impurities which reach the plasma. The ratios of Ohmic power are closer to a factor of two, so wall heating is not as much greater as magnetic power, but even that factor of two may cause serious wall interactions. This could be locally enhanced by azimuthal asymmetries associated with the large level of magnetic turbulence.

Effects such as these are difficult to model and require experimental study. They indicate the importance of early studies of the effect of buildup rate, and suggest that observation of impurities is important in the confined plasma, the edge plasma, and the gun. 


\section{Acknowledgement}

DH would like to thank Drs. Jim Crotinger and Don Pearlstein of the MFE group at the Lawrence Livermore National Laboratory for their help in using corsica. Many thanks also go to the staff at the MFE group at the LLNL for their hospitality.

\section{References}

[1] T. K. Fowler and D. D. Hua, Jour. of Fusion Energy, 14181 (1996).

[2] J. B. Taylor, Rev. Mod. Phys. 58, 741 (1986).

[3] A. B. Rechester and M. N. Rosenbluth, Phys. Rev. Lett. 40, 38 (1978).

[4] F. J. Wysocki, J. C. Fernandez, I. Henins, T. R. Jarboe and G. J. Marklin, Phys. Rev. Lett. 65, 40 (1994).

[5] J. A. Crotinger, L. LoDestro, L. D. Pearlstein, A. Tarditi, T. A. Casper and E. B. Hooper, "Corsica: A Comprehensive Simulation of Toroidal Magnetic-Fusion Devices; Final Report to the LDRD Program." UCRL-ID-126284, (1997). 


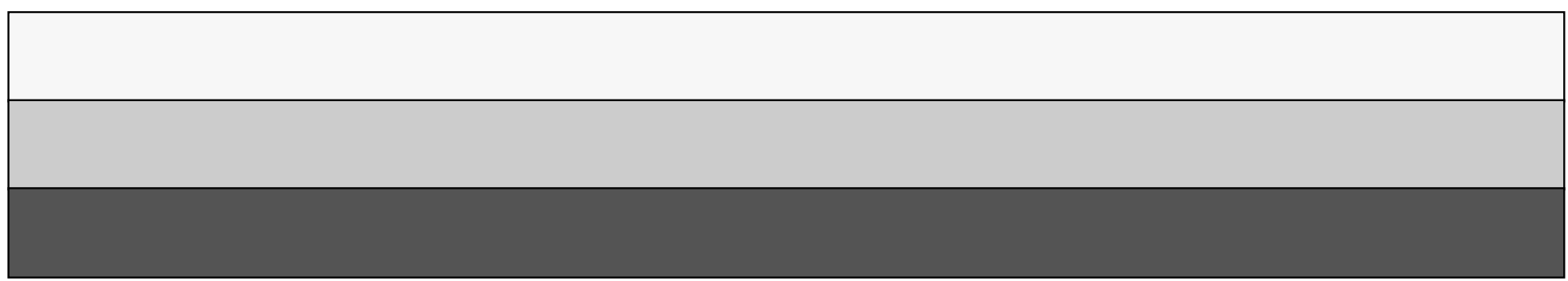

